\title{
Simulation analysis of efficiency and stability for vehicle's ABS control on combined steering and braking maneuvers
}

\author{
Feng YU*, and Jun XIE \\ College of Mechanical Engineering, Chongqing Vocational Institute of Engineering, Chongqing, \\ 402260, China
}

\begin{abstract}
Eight degrees of freedom vehicle model was established. Using the method of fuzzy control, the ABS control algorithm was designed based on slip ratio. Simulation analysis was done at speed of $15 \mathrm{~m} / \mathrm{s}, 20 \mathrm{~m} / \mathrm{s}$, $25 \mathrm{~m} / \mathrm{s}$ under turning braking. The results show that the vehicle braking performance and vehicle stability at middle or low speed was improved by using the ABS controller, but qualitative analysis shows that phenomenon of vehicle instability was appeared at high-speed conditions. The turning braking stability under ABS controller was judged quantificationally by the stability judging formula. The results show that the requirements of stability control could not meet with only Anti-lock Braking System.
\end{abstract}

\section{Preface}

As velocity of vehicles continues to increase, the demand for active safety is subsequently higher and higher. Curved braking is a common yet complicated working condition when load transfer would cause large lateral slide, imposing stricter requirements on vehicle active control.

At present, there are many researches on anti-lock braking systems (ABS) at home and abroad, which mainly focus on the design of control algorithms in straight-line motion [1-4]. In addition, some scholars have conducted simulation studies on ABS control during curved braking, most of which however are limited to the improvement of the braking efficiency of ABS systems, ignoring the investigations on vehicle stability when there is only ABS control at different velocity. [5]

In view of curved braking condition, combined Pacejka "magic formula" tire model was selected for the establishment of an 8-DOFs vehicle dynamics model. Based on the fuzzy control strategy, an ABS control algorithm related to the slip ratio was designed. Simulations of braking efficiency and stability at three different initial vehicle speeds were performed. The results show that the designed ABS control system can effectively improve the braking performance. At the same time, the stability of the vehicle can be also improved at low and medium speeds. However, at high speeds, the vehicle is more likely to lose stability when there is only ABS braking control available. Meanwhile, this is verified in quantitative perspective using stability criterion.

* Corresponding author: cquyf@163.com 


\section{The establishment of simulation model}

\subsection{The establishment of dynamic model}

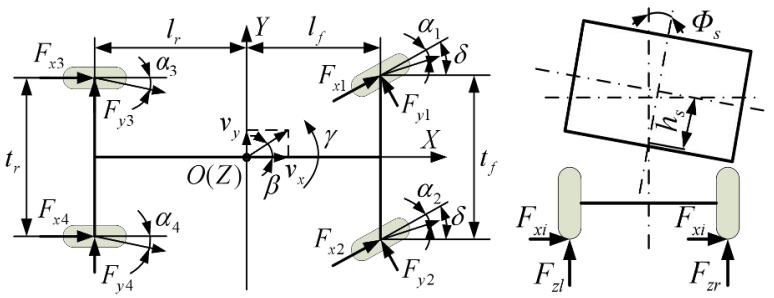

Fig. 1. Four-wheel vehicle model with eight degrees of freedom.

To better describe the dynamic characteristics of the vehicle, a four-wheel 8 DOFs vehicle dynamics model was established as shown in Figure 1, including the rotation of four wheels as well as yaw, roll, lateral and longitudinal movements of car body.

Ignoring the impact of air resistance and tire rolling resistance, the basic equations of vehicle movement are as follows:

$$
\begin{aligned}
& \sum \mathrm{F}_{\mathrm{xi}}=\mathrm{m}\left(\dot{\mathrm{v}}_{\mathrm{x}}-\mathrm{v}_{\mathrm{y}} \gamma\right) \\
& \sum \mathrm{F}_{\mathrm{yi}}=\mathrm{m}\left(\dot{\mathrm{v}}_{\mathrm{y}}+\mathrm{v}_{\mathrm{x}} \gamma\right) \quad(\mathrm{i}=1,2,3,4) \\
& \mathrm{I}_{\mathrm{z}} \dot{\gamma}=\sum \mathrm{M}_{\mathrm{z}} \\
& \begin{array}{c}
\mathrm{I}_{\mathrm{x}} \dot{\mathrm{p}}=\sum_{\mathrm{wi}} \mathrm{M}_{\mathrm{x}}+\mathrm{m}_{\mathrm{s}} \mathrm{h}_{\mathrm{s}}\left(\dot{\mathrm{v}}_{\mathrm{y}}+\mathrm{v}_{\mathrm{x}} \gamma\right) \\
\dot{\mathrm{w}}_{\mathrm{wi}}=\mathrm{F}_{\mathrm{xi}} \mathrm{R}-\mathrm{T}_{\mathrm{bi}}
\end{array} \\
& \sum \mathrm{F}_{\mathrm{xi}}=\left(\mathrm{F}_{\mathrm{x} 1}+\mathrm{F}_{\mathrm{x} 2}\right) \cos \delta-\left(\mathrm{F}_{\mathrm{y} 1}+\mathrm{F}_{\mathrm{y} 2}\right) \sin \delta+\mathrm{F}_{\mathrm{x} 3}+\mathrm{F}_{\mathrm{x} 4} \\
& \sum \mathrm{F}_{\mathrm{yi}}=\left(\mathrm{F}_{\mathrm{y} 1}+\mathrm{F}_{\mathrm{y} 2}\right) \cos \delta+\left(\mathrm{F}_{\mathrm{x} 1}+\mathrm{F}_{\mathrm{x} 2}\right) \sin \delta+\mathrm{F}_{\mathrm{y} 3}+\mathrm{F}_{\mathrm{y} 4} \\
& \sum \mathrm{M}_{\mathrm{z}}=1_{\mathrm{f}}\left(\mathrm{F}_{\mathrm{y} 1}+\mathrm{F}_{\mathrm{y} 2}\right) \cos \delta-1_{\mathrm{r}}\left(\mathrm{F}_{\mathrm{y} 3}+\mathrm{F}_{\mathrm{y} 4}\right)+\frac{\mathrm{t}_{\mathrm{f}}}{2}\left(\mathrm{~F}_{\mathrm{y} 1}-\mathrm{F}_{\mathrm{y} 2}\right) \sin \delta- \\
& \frac{\mathrm{t}_{\mathrm{f}}}{2}\left(\mathrm{~F}_{\mathrm{x} 1}-\mathrm{F}_{\mathrm{x} 2}\right) \cos \delta+1_{\mathrm{f}}\left(\mathrm{F}_{\mathrm{x} 1}+\mathrm{F}_{\mathrm{x} 2}\right) \sin \delta-\frac{\mathrm{t}_{\mathrm{r}}}{2}\left(\mathrm{~F}_{\mathrm{x} 3}-\mathrm{F}_{\mathrm{x} 4}\right) \\
& \sum \mathrm{M}_{\mathrm{x}}=-\left(\mathrm{k}_{\mathrm{f}}+\mathrm{k}_{\mathrm{r}}\right) \Phi_{\mathrm{s}}-\left(\mathrm{c}_{\phi_{\mathrm{f}}}+\mathrm{c}_{\phi_{\mathrm{r}}}\right) \mathrm{p}+\mathrm{m}_{\mathrm{s}} \mathrm{g} \mathrm{h}_{\mathrm{s}} \sin \Phi_{s}
\end{aligned}
$$

Some interpretations for the formula are: $\sum F_{x i}, \sum F_{y i}$ is longitudinal and transverse joint force of tires; $M_{x}, M_{z}$ is moment of force around $\mathrm{X}$ and $\mathrm{Z}$ axis; $\mathrm{m}, \mathrm{m}_{\mathrm{s}}$ is curb weight and suspended mass; $v_{x}, v_{y}$ is velocity along $\mathrm{X}$ and $\mathrm{Y}$ direction; $\dot{v}_{x}, \dot{v}_{y}$ is acceleration along $\mathrm{X}$ and $\mathrm{Y}$ direction; $l_{f}, l_{r}$ is the distance between centroid and front and rear axles; $t_{f}, t_{r}$ is front and rear wheelbase; $\delta$ is steering angle of steering wheels; $\mathrm{g}$ is gravitational acceleration; $\gamma, \dot{\gamma}$ is yaw angular speed and yaw angular acceleration; $\mathrm{p}, \dot{\mathrm{p}}$ is heeling angular speed and heeling angular acceleration; $\varpi_{w i}$ is angular speed of wheels $(i=1,2,3,4) ; I_{w i}$ is moment of wheels( $\left.i=1,2,3,4\right) ; T_{b i}$ is braking torque of brakes $(i=1,2,3,4) ; F_{x i}, F_{y i}$ is longitudinal and lateral force of wheels $(i=1,2,3,4) ; k_{f}, k_{r}$ is rolling angle stiffness of front and rear axles; $I_{x}, I_{z}$ is suspended mass's rotational moment around $\mathrm{X}$ and $\mathrm{Z}$ axis; $c_{\phi_{f}}, c_{\phi_{r}}$ is rolling angle damping of front and rear axles; $R$ is static radius; $\Phi_{\mathrm{s}}$ is rolling angle; $h_{s}$ is distance between centroid and rolling center. Parameter values are shown as Table 1 with standard units. 
Table 1. Parameters for simulation.

\begin{tabular}{|c|c|c|c|c|}
\hline$m$ & $m_{s}$ & $m_{u}$ & $L$ & $l_{f}$ \\
\hline 1370 & 1228 & 142 & 2.64 & 1.26 \\
\hline$l_{r}$ & $t_{f}$ & $t_{r}$ & $h_{c}$ & $h_{s}$ \\
\hline 1.38 & 1.535 & 1.531 & 0.48 & 0.36 \\
\hline$h_{u}$ & $I_{z}$ & $I_{x}$ & $I_{w i}$ & $R$ \\
\hline 0.28 & 1600 & 720 & 2.1 & 0.31 \\
\hline$k_{f}$ & $k_{r}$ & $c_{\varphi_{s}}$ & $c_{\varphi_{r}}$ & \\
\hline 38400 & 31420 & 1756 & 1756 & \\
\hline
\end{tabular}

\subsection{The establishment of tire model}

Pacejka "Magic Formula" model of nonlinear tire in combined condition can accurately reflect the tire characteristics during turning braking [6-7], therefore Pacejka "magic formula" tire model was selected during the modeling process. Neglect the influence of the positive torque; the input of the tire model is the normal reaction force, the tire slip angle and the slip ratio $\lambda$; the output is the longitudinal force $F_{x}$ and the lateral force $F_{y}$.

$$
\begin{gathered}
\mathrm{F}_{\mathrm{x}}=\left(\sigma_{\mathrm{x}} / \sigma\right) \mathrm{F}_{\mathrm{x} 0} \\
\mathrm{~F}_{\mathrm{y}}=\left(\sigma_{\mathrm{y}} / \sigma\right) \mathrm{F}_{\mathrm{y} 0} \\
\mathrm{~F}_{\mathrm{x} 0}=\mathrm{D}_{\mathrm{x}} \cdot \sin \left(\mathrm{C}_{\mathrm{x}} \cdot \arctan \left(\mathrm{B}_{\mathrm{x}} \cdot \phi_{\mathrm{x}}\right)\right) \\
\mathrm{F}_{\mathrm{y} 0}=\mathrm{D}_{\mathrm{y}} \cdot \sin \left(\mathrm{C}_{\mathrm{y}} \cdot \arctan \left(\mathrm{B}_{\mathrm{y}} \cdot \phi_{\mathrm{y}}\right)\right) \\
\sigma=\sqrt{\sigma_{\mathrm{x}}^{2}+\sigma_{\mathrm{y}}^{2}} \\
\sigma_{\mathrm{x}}=\lambda /(1-\lambda) ; \sigma_{\mathrm{y}}=\tan \alpha /(1-\lambda)
\end{gathered}
$$

Here, $F_{x 0}, F_{y 0}$ is longitudinal force under pure longitudinal sliding motion and transverse force under pure side sliding motion; $\sigma$ is combined slip ratio; $\mathrm{D}_{\mathrm{x}}, \mathrm{D}_{\mathrm{y}}, \mathrm{C}_{\mathrm{x}}$, $\mathrm{C}_{\mathrm{y}}, \mathrm{B}_{\mathrm{x}}, \mathrm{B}_{\mathrm{y}}$ is constant of Pacejka "Magic Formula" model of nonlinear tire in combined condition; $\phi_{\mathrm{x}}, \phi_{\mathrm{y}}$ is normal force and function of slip angle.

In this tire model, the load transfer caused by vehicle braking, cornering, and roll has been taken into consideration in the normal force of the tire. The slip angle during the motion is estimated by equation (12) [8].

$$
\left\{\begin{array}{l}
\alpha_{1}=\arctan \left(\frac{\mathrm{v}_{\mathrm{y}}+1_{\mathrm{f}} \cdot \gamma}{\mathrm{v}_{\mathrm{x}}-\mathrm{t}_{\mathrm{f}} / 2 \cdot \gamma}\right)-\delta ; \alpha_{2}=\arctan \left(\frac{\mathrm{v}_{\mathrm{y}}+1_{\mathrm{f}} \cdot \gamma}{\mathrm{v}_{\mathrm{x}}+\mathrm{t}_{\mathrm{f}} / 2 \cdot \gamma}\right)-\delta \\
\alpha_{3}=\arctan \left(\frac{\mathrm{v}_{\mathrm{y}}-1_{r} \cdot \gamma}{\mathrm{v}_{\mathrm{x}}-\mathrm{t}_{r} / 2 \cdot \gamma}\right) ; \alpha_{4}=\arctan \left(\frac{\mathrm{v}_{\mathrm{y}}-1_{r} \cdot \gamma}{\mathrm{v}_{\mathrm{x}}+\mathrm{t}_{r} / 2 \cdot \gamma}\right)
\end{array}\right.
$$

\section{ABS control strategy during curved braking}

As the ABS control strategy of the automobile, we generally aim to achieve a good lateral force and a high braking intensity through the control of wheel slip ratio. Fuzzy control is an intelligent control method which can simulate human brain's fuzzy inference mechanism and dynamically regulate the system according to certain control rules. With good 
robustness and strong practicality, this method is independent from mathematical models[9].

The fuzzy controller's control rules are generally based on human's manual control rules, while the basic information acquired from the manual control is error, the error variation and the output of the control action, therefore the error E and the error change EC is generally selected as the fuzzy controller input variable, and $U$ for the output.

The process of fuzzy control is to blur the error and error change, and to defuzzify the control variable at the same time. Assume the basic domain of error is $[-e, e]$, the basic domain of error variation is $[-e c, e c]$, and the basic domain of control volume is $[-u, u]$, where the value of $e, e c, u$ is determined by the actual measured value.

Assume the discourse domain for the fuzzy subsets of error variables is: $\{-\mathrm{N},-\mathrm{N}+1, \ldots, 0, \ldots, \mathrm{N}-1, \mathrm{~N}\}$; the discourse domain for the fuzzy subsets of error variation variables is: $\{-\mathrm{M},-\mathrm{M}+1, \ldots, 0, \ldots, \mathrm{M}-1, \mathrm{M}\}$; and the discourse domain for the fuzzy subset of the control variable is: $\{-\mathrm{L},-\mathrm{L}+1, \ldots, 0, \ldots, \mathrm{L}-1, \mathrm{~L}\}$, generally the range is $\mathrm{N} \geq 6, \mathrm{M} \geq 6, \mathrm{~L} \geq 7$.

To perform the fuzzification process, the input variables should be transformed from the basic discourse domain to the corresponding discourse domain of fuzzy set. At the same time, the input variables must be multiplied by corresponding quantification factor. The quantification factor $K_{e}$ of the error and the quantification factor $K_{e c}$ of the error change are determined by Equation (13) and Equation (14) respectively:

$$
\begin{aligned}
& K_{e}=N / e \\
& K_{e c}=M / e c
\end{aligned}
$$

Similarly, the scale factor is determined by Equation (15) for the defuzzification of the controller output:

$$
K_{u}=u / L
$$

In the actual design, the expected slip ratio is $\lambda_{o p t}$, the slip rate deviation $e$ and the change rate of deviation $\dot{e}$ are defined as shown in formula (16):

$$
\left\{\begin{array}{l}
e=\lambda-\lambda_{\text {opt }} \\
\dot{e}=\frac{\mathrm{d} e}{\mathrm{~d} t}=\frac{\mathrm{d}\left(\lambda-\lambda_{\text {opt }}\right)}{\mathrm{d} t}
\end{array}\right.
$$

The discourse domain is defined as $[-6,6]$. The respective linguistic variables are divided into 6 sub items: \{negative big (NB), negative medium (NM), zero (ZE), positive small (PS), positive medium (PM), positive big (PB) $\}$. The change of wheel brake torque $\Delta T_{b}$ is the controlled output, whose discourse domain and membership function distribution of subitem in the discourse domain is the same as deviation and the change rate of the slip rate.

Based on the relationship between the input and output variables in the actual braking process of the vehicle, a fuzzy control logic rule set is produced, as shown in Table 2 . There is a total of 49 rules in the fuzzy control strategy. Here we take the first one as an example. When the error is NB and the error variation is NB, the controlled output is PB. The physical meaning indicated is that the increase in the control torque reaches positive maximum when the slip error is negative big and continues to increase at the maximum rate. Mamdani fuzzy inference is adopted as the fuzzy methodology, and the centroid method for controller defuzzification. 
Table 2. Fuzzy control rules of ABS.

\begin{tabular}{|c|c|c|c|c|c|c|c|}
\hline$\dot{e}$ & $N B$ & $N M$ & $N S$ & $Z E$ & $P S$ & $P M$ & $P B$ \\
\hline$N B$ & $P B$ & $P B$ & $P B$ & $P B$ & $P M$ & $Z E$ & $Z E$ \\
\hline$N M$ & $P B$ & $P B$ & $P B$ & $P B$ & $P M$ & $Z E$ & $Z E$ \\
\hline$N S$ & $P M$ & $P M$ & $P M$ & $P M$ & $Z E$ & $N S$ & $N S$ \\
\hline$Z E$ & $P M$ & $P M$ & $P S$ & $Z E$ & $N S$ & $N M$ & $N M$ \\
\hline$P S$ & $P S$ & $P S$ & $Z E$ & $N M$ & $N M$ & $N M$ & $N M$ \\
\hline$P M$ & $Z E$ & $Z E$ & $N M$ & $N B$ & $N B$ & $N B$ & $N B$ \\
\hline$P B$ & $Z E$ & $Z E$ & $N M$ & $N B$ & $N B$ & $N B$ & $N B$ \\
\hline
\end{tabular}

\section{Efficiency analysis of ABS control during curved braking}

To analyze the ABS control efficiency during curved conditions, the simulation analysis of ABS control at different initial velocity was carried out.

The simulation conditions are as follows: from 0 to $0.5 \mathrm{~s}$, the front wheel rotation angle is uniformly raised to $0.04 \mathrm{rad}$ and then hold [10]. The braking starts at $1 \mathrm{~s}$ with the road adhesion coefficient as 0.75 and the controlled target slip ratio as 0.15 . The fuzzy control error, the quantification factor of the error variation and the quantification factor of the output torque variation are 16,2 and 8000 respectively.

For the initial velocity of $15 \mathrm{~m} / \mathrm{s}, 20 \mathrm{~m} / \mathrm{s}$, and $25 \mathrm{~m} / \mathrm{s}$, the simulation results of turning ABS control are shown in Figures 2, 3, and 4.

From Figure 2, the slip ratio control of each wheel is effective at low velocity, with even change in the velocity and little change in the control torque. The magnitude of the braking torque of each wheel is mainly determined by the different loads of the wheels, therefore, the front wheel needs a larger braking torque owing to its relative larger load. Similarly, for the left and right wheels, the outer wheels require a larger braking torque.
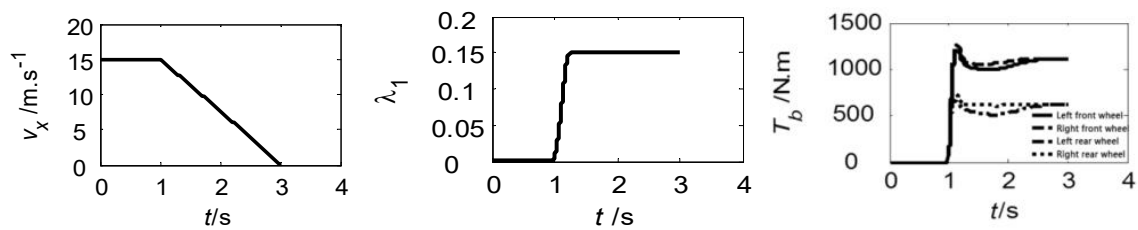

(a) Vehicle longitudinal velocity (b) Slip rate of left-front wheel (c) Braking torque of each wheel Fig. 2. Simulation results with initial speed $15 \mathrm{~m} / \mathrm{s}$.
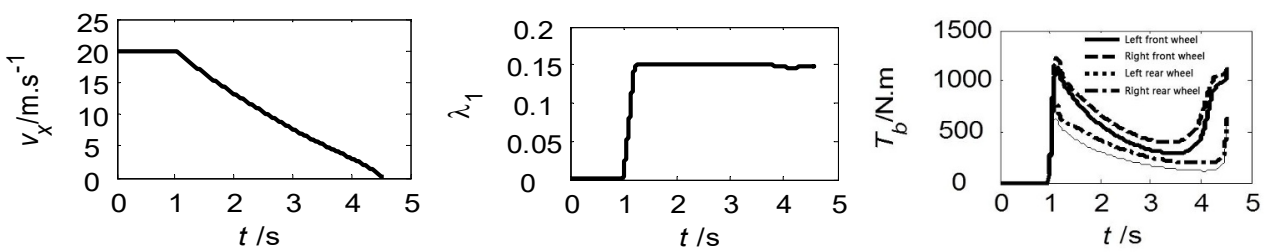

(a) Vehicle longitudinal velocity (b) Slip rate of left-front wheel (c) Braking torque of each wheel Fig. 3. Simulation results with initial speed $20 \mathrm{~m} / \mathrm{s}$.

From Figure 3, we can see that when the initial speed is $20 \mathrm{~m} / \mathrm{s}$, the wheel slip rate can still be well controlled at 0.15 . However, there is some fluctuation in later control stage, it is mainly because the absolute velocity is smaller afterwards and the change of control torque will result into the fluctuation of the slip rate. At the same time, the profile of vehicle speed changes as well as the braking torque is concave. This is mainly because the longitudinal force provided by the ground to the vehicle is smaller in the middle stage, thus the speed reduced slowly, and the corresponding control torque is small. 

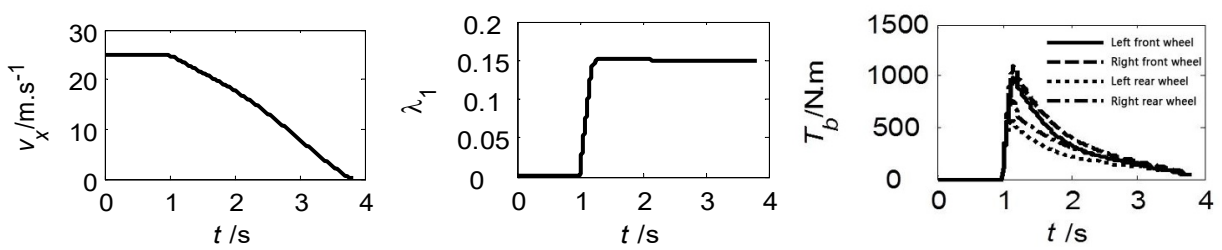

(a) Vehicle longitudinal velocity (b) Slip rate of left-front wheel (c) Braking torque of each wheel Fig. 4. Simulation results with initial speed $25 \mathrm{~m} / \mathrm{s}$.

It can be seen from Figure 4 that when the initial speed reaches $25 \mathrm{~m} / \mathrm{s}$, the effect of slip rate control is still reasonable. But the profile of vehicle speed changes is convex, and the braking torque continues to decrease after rapid rise. The decrease of torque in the rear section is mainly due to the reduction of the longitudinal force provided by each wheel, which means only a small control torque is required to reach an ideal value for the wheel slip ratio.

Based on Figures 2, 3 and 4, the higher the initial braking speed of the vehicle is, the smaller the braking control torque would be needed. The difference in braking control torque is relatively large especially in the middle and later stages of braking, which is mainly due to the increase in the side slip angle of each wheel during the braking process.

\section{Stability analysis of ABS control during curved braking}

Figure 5 shows the lateral acceleration and lateral slip velocity obtained from the simulation. It can be seen from Figure 5 that the lateral acceleration of the vehicle shows a sharp rise in the first second and rapidly decreases. Although it gradually increases thereafter, the peak value does not exceed the peak value before $1 \mathrm{~s}$, mainly due to the change of lateral force provided by the tire during braking and the change in the side slip angle of the wheel. After the braking begins, the lateral force of the tire decreases with the increase of the slip rate. The slip rate rises rapidly, so that the lateral acceleration rapidly decreases.

When the initial speed is relatively high, the lateral acceleration decreases sharply in the later stage, resulting in a drastic decrease of the lateral force provided by the wheels. Obviously, there is a side-slip and tail-flicking.

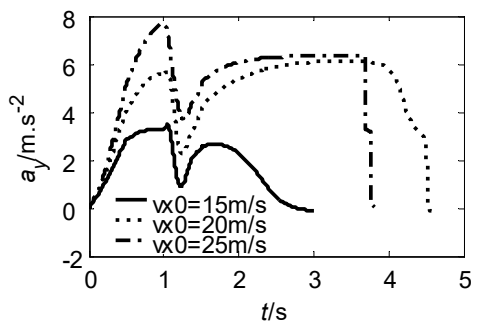

(a) lateral acceleration

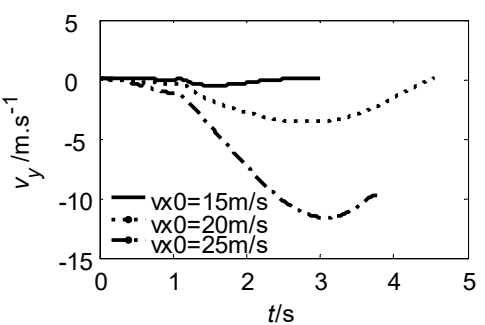

(b) lateral slip velocity

Fig. 5. Running state comparisons between the simulations with different initial speeds.

As can be seen in connection with Figures 3, 4 and 5, braking time with the initial speed of $25 \mathrm{~m} / \mathrm{s}$ is shorter than that with the initial speed of $20 \mathrm{~m} / \mathrm{s}$, which means that the vehicle has completely lost control.

Studies by Japanese scholars Ken Koibuchi and Masaki Yamamoto et al. show that whether the vehicle is in a stable state or not is determined by the side slip angle of the vehicle centroid and its angular velocity, and the stability determination formula [11] is proposed. At the same time, Akihiko SHIMURA has presented a criterion for the stability of sideslip angle through neural network and genetic algorithm [12]: 


$$
|12.58 * \beta+4.53 * \dot{\beta}|<1.0
$$

Using relevant parameters obtained from simulations, together with this stability criterion, we can quantitatively evaluated the vehicle stability during curved braking.

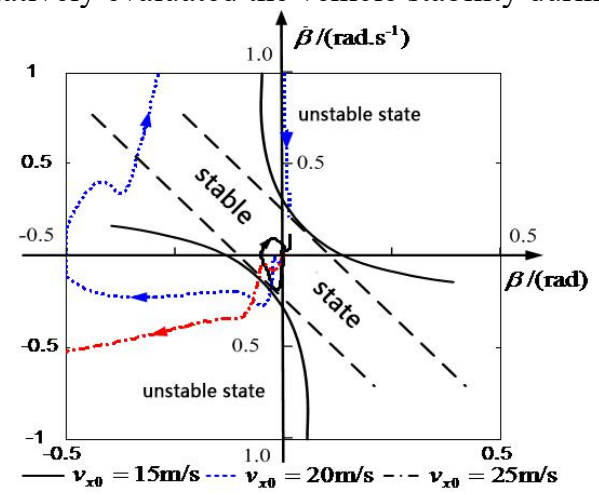

Fig. 6. $\beta-\dot{\beta}$ phase trace with $\mathrm{ABS}$ control on combined steering and braking maneuvers.

Figure 6 shows the $\beta-\dot{\beta}$ phase plane plot of ABS control at three different initial vehicle speeds. According to the stability criteria: when the initial speed is $15 \mathrm{~m} / \mathrm{s}$, the vehicle with ABS control is always in a stable state; when the initial speed is $20 \mathrm{~m} / \mathrm{s}$ and $25 \mathrm{~m} / \mathrm{s}$, the vehicle quickly loses stability even if there is ABS control. Moreover, the degree of instability of the vehicle becomes more serious as the initial speed increases.

\section{Conclusion}

For the curved braking working conditions, the vehicle's braking efficiency and stability with ABS control at three different velocities are simulated and analyzed. The main conclusions are drawn as follows:

(1) For different initial vehicle speeds, the ABS fuzzy control algorithm can get better control over the target slip ratio and confine it to 0.15 or so, which shows that the fuzzy control algorithm has effective control on anti-lock braking of vehicles.

(2) During the curved braking, ABS control can improve the braking efficiency as well as improve the stability to a certain extent. However, qualitative analysis shows that the vehicle would easily lose stability when the vehicle speed is high. Moreover, as the initial speed increases, loss of stability tends to be more severe.

(3) Based on the stability criteria, the stability during curved braking at different velocities when there is only ABS control available is quantitatively analyzed. It is further verified that vehicles during curved braking would easily lose stability when the initial velocity is high. In addition, this will become more severe as the vehicle speed increases, which calls for further stability control strategies.

\section{Funding}

The author(s) disclosed receipt of the following financial support for the research, authorship, and/or publication of this article: This study was supported by Chongqing Municipal Education Commission Science and Technology Project (No.KJ1603207 \& No.KJQN201803408). 


\section{References}

1. Zhang Xin,Lou Yuanyuan,Yang Xiufang. Study of control logic for automobile anti-lock braking system[C]. ICICTA 2008:International Conference on Intelligent Computation Technology and Automation, Changsha, October 20-22, 2008. 493 497

2. Li Rui,Zheng Taixiong, Li Yinguo. Hierarchical Intelligent Control of Automobile Anti-Lock Braking System [J]. Journal of Mechanical Engineering, 2007, 43(8):135 140

3. Sun Yuedong,Zhou Ping,Kang Min,Li Chun. Simulation of Automotive ABS Based on PSD Control [J]. Transactions Of The Chinese Society For Agricultural Machinery, 2007, 38(2): 39 41

4. ZHANG Jianlong,YIN Chengliang,ZHANG Jianwu. Design and Analysis of Electro-mechanical Hybrid Anti-lock Braking System for Hybrid Electric Vehicle Utilizing Motor Regenerative Braking[J].. Chinese Journal of Mechanical Engineering, 2009, 20(1): 42 49

5. ZANG Huai-quan,LI Zhen-peng,SHI Qing-zhou. Application of Double Model Controller in Swerve Brake System [J]. Journal Of System Simulation, 2006, 18(7):1897 1901

6. E. Bakker,H. B Pacejka, L. Lidner. A new Tire Model with an Application in Vehicle Dynamics Studies[C]. SAE Paper 890087: 101 113

7. E. Bakker,H. B Pacejka. Tyre Modelling for Use in Vehicle Dynamics Studies[C]. SAE Paper 870421: 2.190 2.204

8. Li Jun,Zhang Jianwu. Investigation to The Rapid Development of Vehicle Abs Control System by Using Hardware-In-The-Loop Simulation Technology [J]. Chinese Journal of Mechanical Engineering, 2002, 38(7): 84 90

9. Liu Shuguang,Wei Junmin,Zhu Zhichao. Fuzzy Control Technique[M]. Beijing: China Textile \& Apparel Press, 2001

10. Xu Zhongming,Yu Feng,Zhang Zhifei. Co-simulation of ABS for Vehicles under Turning Braking Based on ADAMS and Simulink[J]. China Mechanical Engineering, 2009, 20(7): 877 881

11. Ken Koibuchi,Masaki Yamamoto,Yoshiki Fukada et al.. Vehicle Stability Control in Limit Cornering by Active Brake[C]. SAE Paper 960487: 555 565

12. Akihiko SHIMURA,Kazuo YOSHIDA. Rule Extraction with Connection Forgetting from Neural Network DYC Controller[C]. AVEC'02 20024575: 2837 2842 